\title{
Intervenção da fisioterapia na síndrome de Menkes: relato de caso
}

\section{Intervention of physiotherapy in Menkes syndrome: case report}

(DBianca Kons dos Santos ${ }^{1}$, DBianca Dana Horongozo Itaborahy ${ }^{1}$, Fabiula Joanita da Mata Belém ${ }^{1}$, (1) Camila Isabel Santos Schivinski ${ }^{1}$

\section{RESUMO}

Objetivo: Descrever a intervenção da fisioterapia motora e respiratória no caso de uma criança com Síndrome de Menkes. Método: Relato de caso, com base em registros retrospectivos, no qual são apresentados dados referentes ao acompanhamento fisioterapêutico de uma criança com o diagnóstico de Síndrome de Menkes. Os dados foram obtidos por meio do prontuário, entrevista com familiares e informações dos profissionais envolvidos. O referido paciente foi encaminhado para assistência fisioterapêutica aos 5 meses de vida, devido ao quadro de pneumonia com presença de atelectasia, associado as manifestações típicas da Síndrome, sendo então acompanhado por um período de 04 meses. Foram realizados 76 atendimentos, de um total de 91 agendamentos, os quais incluíram fisioterapia motora e respiratória, sendo aplicados métodos, técnicas, manuseios e posturas, para estimulação do desenvolvimento neuropsicomotor, e realizadas técnicas e recursos fisioterapêuticos para desobstrução e reexpansão pulmonar. Resultados: A cada sessão, a criança apresentou evidente melhora imediata no padrão e tipo respiratório, na frequência respiratória, na ausculta pulmonar e nos sinais de desconforto respiratório. Além disso, a estimulação motora e manutenção do quadro músculo esquelético, impediram agravos e deformidades. Segundo relato da mãe, a criança mostrava-se menos agitada após as sessões, com melhora no padrão e conforto respiratório, o que impactou de forma positiva na sua qualidade de vida. Conclusão: A fisioterapia motora e respiratória se apresentam como terapêuticas favoráveis para condição de saúde geral de pacientes com Síndrome de Menkes e novos estudos devem ser conduzidos no sentido de elucidar essa intervenção, com amostras maiores.

Palavras-chaves: Síndrome dos Cabelos Torcidos, Predisposição Genética para Doença, Modalidades de Fisioterapia, Criança

\begin{abstract}
Objective: To describe the intervention of motor and respiratory physiotherapy in the case of a child with Menkes Syndrome. Method: Case report based on retrospective registers in which data are presented regarding the physical therapy accompaniment of a child with the diagnosis of Menkes Syndrome. The data were obtained based on the records of the child's chart, interview with relatives and information of the professionals involved. The patient was referred for physiotherapeutic assistance at 5 months of age, due to the presence of atelectasis pneumonia associated with the typical manifestations of Menkes' Syndrome, followed by a period of 4 months. A total of 76 appointments were performed, including motor and respiratory physiotherapy, and methods, techniques and manipulations were used to stimulate neuropsychomotor development, as well as techniques and physiotherapeutic resources were used to clear and reexpans the lungs. Results: At each session, the child showed evident immediate improvement in respiratory pattern and type, respiratory rate, pulmonary auscultation, and signs of respiratory discomfort. In addition, the motor stimulation and maintenance of the skeletal muscle of the child, prevented injuries and deformities. According to the mother's report, the child was less agitated after the sessions, with improved breathing pattern and comfort, which positively impacted his quality of life. Conclusion: Motor and respiratory physiotherapy are presented as favorable therapies for the general health condition of patients with Menkes-Syndrome, and further studies should be conducted to elucidate this intervention in a bigger sample.
\end{abstract}

Keywords: Menkes Kinky Hair Syndrome, Genetic Predisposition to Disease, Physical Therapy Modalities, Child

\section{Como citar}

Santos BK, Itaborahy BDH, Belém FJM, Schivinski CIS. Intervenção da fisioterapia na síndrome de Menkes: relato de caso. Acta Fisiatr. 2020;27(1):58-63.

\section{(c) (1) (5) (2)}

C) 2020 by Acta Fisiátrica

Este trabalho está licenciado com uma licença Creative Commons - Atribuição 4.0 Internacional 


\section{INTRODUÇÃO}

A Síndrome de Menkes (SM) é uma condição congênita rara, causada por mutações genéticas ligadas ao gene ATP7A, ${ }^{1,2,3}$ cujas alterações ocasionam uma desordem do metabolismo de cobre. As ações desempenhadas pelas enzimas que dependem deste elemento são prejudicadas, o que causa sinais característicos da SM. ${ }^{1,4}$ Durante os primeiros meses de vida, a maioria das crianças acometidas não apresentam sinais, apenas alterações inespecíficas, o que leva a grande dificuldade no diagnóstico precoce, resultando em manifestações graves. ${ }^{3} \mathrm{O}$ diagnóstico é baseado em achados bioquímicos como baixos níveis séricos de ceruloplasmina e cobre, e às próprias características clínicas. ${ }^{1,3}$ Suspeita-se da SM quando a criança apresenta regressão ou atraso no desenvolvimento neuropscimotor (DNPM), sintomas degenerativos, alterações do tecido conjuntivo, hipopigmentação e anormalidades na estrutura do cabelo. ${ }^{1}$

Na sua forma clássica, manifesta-se com maior frequência em meninos, apresenta alterações neurológicas graves e prognóstico fatal em torno dos três anos de vida. ${ }^{1}$ Sua incidência estimada é muito baixa, por isso é considerada rara. ${ }^{1,5} \mathrm{Em}$ virtude das suas características, a indicação da fisioterapia (Ft) é de extrema importância, pois irá atuar nas atividades motoras e cuidados respiratórios, objetivando um melhor desenvolvimento global da criança e manutenção da sua saúde.

$\mathrm{Na}$ literatura, observa-se escassez de estudos relacionados à atuação da fisioterapia motora (FtM) e respiratória (FtR) na SM. Isso porque se trata de uma síndrome rara, com baixo índice de suspeita ou falta de familiaridade na prática pediátrica, e cujo diagnóstico é difícil. ${ }^{6}$ Essa carência repercute em insegurança no manuseio fisioterapêutico, lacunas quanto à expectativa de evolução, bem como negligência sobre o impacto dessa terapêutica na qualidade de vida do paciente e de sua família. Nesse contexto, esse trabalho tem o objetivo de relatar o caso de um paciente com SM, o qual recebeu acompanhamento fisioterapêutico ambulatorial em uma clínica escola de um centro universitário.

\section{CASO CLÍNICO}

Paciente A.G.R.P., sexo masculino, nasceu em 30/05/2015 com tempo gestacional de 36 semanas, via parto normal, sem intercorrências. Após o nascimento, mãe identificou aspectos diferentes na fisionomia e, principalmente, em relação à característica dos fios de cabelos, mas nenhum profissional de saúde suscitou qualquer suspeita. Dois tios maternos da criança foram a óbito por aborto espontâneo, o que indica forte suspeita da história genética familiar, uma vez que a SM está relacionada com o sexo. Aos 2 meses iniciou as primeiras manifestações, apresentando dois quadros de convulsões com necessidade de internação e acompanhamento com neurocirurgião. Iniciou-se redução progressiva da dosagem de anticonvulsivos, no entanto, frente ao insucesso no controle do quadro, houve necessidade de aumento da medicação.

Aos 5 meses a criança recebeu o diagnóstico da SM e, nesse período, apresentou episódios importantes de refluxo gastroesofágico, complicados por pneumonia aspirativa. Em dezembro de 2015, por meio da ultrassonografia, detectou-se estenose hipertrófica do piloro, esofagite grave e hérnia de hiato, sendo realizada a pilorotomia. Após esta intervenção, a alimentação realizada via oral resultou em novos episódios de regurgitação. A criança evoluiu para desnutrição, sendo prescrita nutrição parenteral prolongada em janeiro de 2016 e, posteriormente, colocação da jejunostomia.

A mãe expõe que o filho passou por diversas intervenções médicas e complicações durante os períodos de internação (crises epiléticas, regurgitações e trombose). Em ocasião de maior gravidade, foi transferida para isolamento e a equipe comunicou a família sobre um prognóstico ruim, com óbito projetado para 24 horas. Sem justificativas, houve melhora do quadro.

Em maio, a mãe observou que o filho "se contorcia", e atribuiu o quadro a dor. Após exame de radiografia, constatou-se compressão do pulmão devido à hérnia de hiato do estômago, sendo realizada cirurgia para correção. No dia seguinte, a ultrassonografia evidenciou presença de pequeno derrame pleural em hemitórax direito, associado à consolidação (Quadro 1).
A criança permaneceu internada e foi submetida a gastrostomia, evoluiu com complicações pulmonares e consequente atelectasia, recebendo alta com indicação de oxigenoterapia. Em junho, paciente apresentou pneumonia, ainda com a presença da atelectasia, e foi indicada a FtR diária, sendo encaminhado para a Clínica Escola de Fisioterapia na Universidade do Estado de Santa Catarina (UDESC) em Florianópolis.

\section{Avaliação fisioterapêutica}

A criança foi avaliada em 08/08/2016, aos 15 meses de vida, e a queixa principal da mãe foi a "necessidade de melhorar a função motora e respiratória". Paciente apresentava diagnóstico de SM e enfermidades associadas: esofagite, hérnia de hiato corrigida, estenose hipertrófica de piloro corrigida, bexiga neurogênica, broncoespasmo, atelectasia em hemitórax esquerdo e pneumonia de repetição. Aplicou-se um instrumento de observação padronizado, elaborado e validado para medida da função motora grossa (GMFM), ao longo do tempo, nas crianças com paralisia cerebral ${ }^{7}$ e também utilizado para avaliar diversas doenças/síndromes neurológicas, ${ }^{8}$ com intuito de quanitificar a caracteristica do quadro motor. Os exames complementares são descritos no Quadro 1.

Conduziu-se a inspeção do paciente, o qual se encontrava no colo da mãe, sonolento e choroso, fazendo uso contínuo de oxigênio através de cânula nasal (fluxo de $1,5 \mathrm{~L} / \mathrm{min}$ ). Todos os achados da avaliação são apresentados no Quadro 2.

\section{Acompanhamento e intervenção fisioterapêutica}

O protocolo de intervenção fisioterapêutica foi aplicado de agosto a dezembro de 2016. As sessões foram realizadas $4 x /$ semana, com duração de até 60 minutos. Com base na avaliação, o diagnóstico e plano fisioterapêutico foram estabelecidos (Quadro 2), sendo elaborado de forma

Quadro 1. Exames complementares

\begin{tabular}{|c|c|c|}
\hline Data & Exame & Laudo \\
\hline Maio de 2016 & $\begin{array}{l}\text { Tomografia de } \\
\text { tórax }\end{array}$ & $\begin{array}{l}\text { Volumosa hérnia hiatal com herniação de todo o estômago para a base do } \\
\text { hemitórax direito. }\end{array}$ \\
\hline Maio de 2016 & $\begin{array}{l}\text { Ultrassonografia } \\
\text { de tórax }\end{array}$ & Derrame pleural direito, somado a consolidação da base pulmonar direita. \\
\hline $\begin{array}{l}\text { Agosto de } \\
2016\end{array}$ & $\begin{array}{l}\text { Radiografia de } \\
\text { tórax }\end{array}$ & $\begin{array}{l}\text { Opacidade heterogênea difusa bilateral pronunciada em hemitórax direito, com } \\
\text { imagem sugestiva de atelectasia em hemitórax esquerdo, com desvio traquea } \\
\text { (Figura 1). }\end{array}$ \\
\hline
\end{tabular}


Quadro 2. Avaliação e diagnóstico fisioterapêutico cinético-funcional

\begin{tabular}{|c|c|}
\hline Achados motores & Achados respiratórios \\
\hline $\begin{array}{l}\text { Sem interação com o ambiente e pessoas, manifestando apatia; comunicação por meio } \\
\text { de gestos e sons, comprometida, sem reações efetivas; movimentos oculares aleatórios e } \\
\text { sinal do pôr do sol; hipotonia generalizada, espasmos musculares e arreflexia; ausência de } \\
\text { realização de tarefas simples (alcance, preensão, rolamentos) e mudanças de posturas; } \\
\text { desalinhamento postural, manutenção da cabeça em rotação para direita, com } \\
\text { cifoescoliose em "C" direita lombo-torácica }\end{array}$ & $\begin{array}{l}\text { Sinais Vitais: FC: } 86 \text { bpm, } \mathrm{FR}: 23 \text { irpm com apneias, } \mathrm{SpO}_{2}: 97 \% \text { (com } 1,5 \mathrm{~L} / \mathrm{min} \mathrm{O}_{2} \text { ), } \mathrm{AP} \text { : MV } \\
\text { presente, com EC, além de RT. Sem tosse espontânea, mas eficaz e produtiva quando } \\
\text { estimulada, sem expectoração; presença de tiragem de fúrcula e intercostal, e batimento } \\
\text { da asa do nariz. Respiração paradoxal com amplitude superficial. Tórax brevelíneo e } \\
\text { pectus carinatum; Ritmo respiratório patológico com apneias, variando de } 1: 2 / 1: 3, \mathrm{com} \\
\text { prolongamento da expiração durante o choro, o qual era frequente }\end{array}$ \\
\hline $\begin{array}{l}\text { Postura prono: Inicia o movimento para elevar a cabeça, porém não consegue manter. } \\
\text { Pouca movimentação, com posição corporal mantida conforme ajuste do terapeuta; } \\
\text { Mantém posição corporal estendida devido a hipotonia; }\end{array}$ & $\begin{array}{l}\text { Presença de secreções brônquicas e em vias aéreas superiores, ventilação pulmonar } \\
\text { inadequada }\end{array}$ \\
\hline \multicolumn{2}{|l|}{$\begin{array}{l}\text { Postura supino: Roda parcialmente a cabeça, porém tende a permanecer com rotação } \\
\text { para direita e devido a própria postura; Possui poucos movimentos de membros superiores } \\
\text { e inferiores; Não alcança a mão na boca e não trás as mãos para linha média. Mantém } \\
\text { posição corporal estendida devido a hipotonia; }\end{array}$} \\
\hline \multicolumn{2}{|l|}{$\begin{array}{l}\text { Postura quatro-apoios: Não permanece sem auxílio do terapeuta e de instrumentos. Não } \\
\text { possui controle cervical. }\end{array}$} \\
\hline \multicolumn{2}{|l|}{$\begin{array}{l}\text { Postura sentada: Não permanece sem auxílio do terapeuta; quando apoiado no tórax do } \\
\text { terapeuta mantém controle de cabeça por } 3 \text { s. Tende a permanecer com inclinação à } \\
\text { E e rotação a D; }\end{array}$} \\
\hline \multicolumn{2}{|l|}{$\begin{array}{l}\text { Postura em pé: Não permanece sem auxílio do terapeuta. É necessário auxiliar no controle } \\
\text { cervical, corporal e MMll }\end{array}$} \\
\hline \multicolumn{2}{|l|}{$\begin{array}{l}\text { GMFM: Dimensões: Deitar e rolar: } 7.84 \% \text {; Sentar: } 5 \% \text {; Engatinhar e ajoelhar: } 0 \% \text {; Em pé: } 0 \% \text {; } \\
\text { Andar, correr e pular } 0 \%\end{array}$} \\
\hline \multicolumn{2}{|l|}{ *Todas as dimensões são pontuadas até $100 \%$} \\
\hline \multicolumn{2}{|l|}{ Score total das dimensões: $2,56 \%$} \\
\hline \multicolumn{2}{|c|}{ Plano de atendimento fisioterapêutico } \\
\hline Objetivos & Condutas \\
\hline Estimular o DNPM & $\begin{array}{l}\text { Mobilizações articulares, dissociação de cinturas; alongamentos; Descarga de peso; } \\
\text { FNP, Mudanças de decúbitos; Estimulação: de transferências de forma passiva, de } \\
\text { diferentes posturas; de tarefas de alcance, preensão e manipulação; Estímulo à simetria e } \\
\text { conscientização corporal; Estímulo visual e auditivo }\end{array}$ \\
\hline Promover desobstrução de VAS & Limpeza nasal; DRR; Aspiração nasotraqueal \\
\hline Promover desobstruçã de vias Aéreas inferiores & $\begin{array}{l}\text { Vibração, vibrocompressão manual; DAA; Expiração lenta e prolongada; AFE (lenta } \\
\text { e rápida); Ginga torácica; Mudanças de posturas; Estímulo de tosse e tosse assistida; } \\
\text { Aspiração nasotraqueal; Empilhamento de ar; Bag squeezing }\end{array}$ \\
\hline Melhorar a ventilação pulmonar & $\begin{array}{l}\text { Bag squeezing; Empilhamento de ar; Compressão- descompressão; Mudanças de } \\
\text { posturas }\end{array}$ \\
\hline Reduzir desconforto respiratório & Apoios (íliocostal; abdominal; tóraco-abdominal) \\
\hline Orientar sobre cuidados no domicílio & $\begin{array}{l}\text { Estimulação sensório-motora e postural; DAA; Cuidados referentes à oxigenioterapia e ao } \\
\text { procedimento de aspiração }\end{array}$ \\
\hline
\end{tabular}

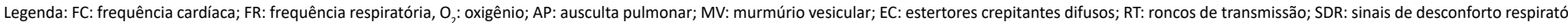

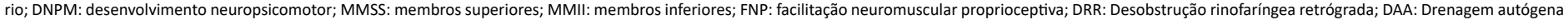
assistida; VAS: vias aéreas superiores; VAI: vias aéreas inferiores;AFE: Aumento do fluxo expiratório

personalizada e de acordo com o quadro clínico do paciente, buscando oferecer a melhora nas capacidades motora e respiratória.

A escolha das técnicas e dos recursos fisioterapêuticos a serem aplicados a cada sessão teve como base a avaliação inicial, a qual era constituída do controle dos parâmetros cardiorrespiratórios, ausculta pulmonar (AP) e sinais de desconforto respiratórios (SDR). Esses parâmetros foram verificados nos atendimentos e anotados em evolução, no início e ao final de cada sessão, para comparação e ajuste das condutas.

O Quadro 3 apresenta dados relacionados a evolução mensal do paciente, de acordo com a avaliação inicial, procedimentos terapêuticos utilizados e resposta terapêutica. Nos casos em que a aspiração foi realizada no domicílio, a mãe preencheu um diário com informações referentes ao procedimento, a qual incluiu: data, horários, quantidade e aspecto e observações. Este registro foi implantado com o intuito de obter informações sobre a rotina domiciliar diária de aspirações e o quadro de saúde do paciente (Quadro 3). Por meio deste registro foi possível observar redução dos procedimentos de aspiração em domicílio nos dias de atendimento fisioterapêutico, e aumento desse procedimento aos finais desemana.

\section{Evolução do paciente e discussão}

Durante o acompanhamento fisioterapêutico de 4 meses, paciente apresentou apenas um episódio de complicação respiratória, tendo sido diagnosticado com pneumonia em outubro de 2016. Nessa ocasião, realizou-se raio-x de tórax, o qual evidenciou melhora do padrão radiológico global (Figuras 1 e 2). Além da melhora no exame radiológico em um período de 2 meses, repercussão positiva imediata da FtR em parâmetros cardiorrepiratórios como: FC, FR, AP e SDR foi constatada (Quadro 4).

No presente relato, a FtM e FtR mostraramse favoráveis para condição de saúde geral do paciente com SM, tanto do ponto de vista motor, quanto respiratório (Quadro 4). Porém, por se tratar de uma síndrome rara, o acompanhamento da Ft nesta enfermidade não tem sido descrito na literatura, o que dificulta comparações com outros casos, e até mesmo um direcionamento para o tratamento fisioterapêutico. No entanto, é possível 
Quadro 3. Evolução fisioterapêutica

\begin{tabular}{|c|c|c|c|c|c|c|}
\hline Período & Técnicas & \multicolumn{2}{|c|}{ Aspirações } & \multicolumn{2}{|l|}{ SCR } & Efeitos imediatos \\
\hline 08/08 à 31/08 & $\begin{array}{l}\text { FtM: AL(C), AL (AL), DP, CC, M, R, } \\
P(S), P(4), E V A \\
\text { FtR: VCM, GT, AFEI, AFEr, ELPr, BS, } \\
\text { EF,A, C/D,A(AB) }\end{array}$ & & & \multicolumn{2}{|c|}{$\begin{array}{l}\text { FC: Variou de } 60 \text { à } 128 \text { bpm } \\
\text { FR: Variou de } 14 \text { à } 32 \text { rpm } \\
\text { SpO2: Variou de } 86 \% \text { à } 99 \% \text {. Ge- } \\
\text { ralmente abaixo de } 95 \% \text { ao início } \\
\text { da sessão } \\
\text { AP: MV } \downarrow \text { com RD em ambos hmt, } \\
\text { além de RT. Às vezes, apresen- } \\
\text { tava EC }\end{array}$} & $\begin{array}{l}\downarrow \text { FR (melhora da taquipneia) } \\
\text { Melhora do padrão e ritmo } \\
\text { respiratório } \\
\uparrow S p O 2 \\
\text { AP ( } \uparrow \text { MV e } \downarrow \text { intensidade dos RA) } \\
\text { Obs: Em uma sessão não houve } \\
\text { diminuição da taquipneia }\end{array}$ \\
\hline 01/09 à 29/09 & $\begin{array}{l}\text { FtM: PC, DP, M, DC, CC, P(DL), P } \\
\text { (S), inclinação de tronco, P(4), P } \\
(O), \quad R E, P(A), A L(C), A L(T), A L \\
(A L), F N P \text { de } M M S S, E V A \\
\text { FtR: } V, V C M, G T, E L P r, D A, A F E I, \\
\text { AFEr, BS, C/D, A, A(AB), A(IC) }\end{array}$ & \multicolumn{2}{|c|}{$\begin{array}{l}\text { Duas sessões sem realizar aspi- } \\
\text { ração } \\
\text { Geralmente: } M \text { e G, vezes F e } \\
\text { outras E. } \\
\text { Sendo C, PR, porém na maior } \\
\text { parte MC }\end{array}$} & \multicolumn{2}{|c|}{$\begin{array}{l}\text { FC: Variou de } 81 \text { à } 150 \text { bpm. } \\
\text { Geralmente na faixa de } 110 \text { à } \\
140 \text { bpm } \\
\text { FR: Variou de } 12 \text { à } 30 \text { rpm } \\
\text { SpO2: Variou de } 86 \% \text { à99\% } \\
\text { Geralmente abaixo de } 95 \% \text { ao } \\
\text { início da sessão } \\
\text { AP: MV } \downarrow \text { em bases, com RD em } \\
\text { ambos hmt, além de RT. Às } \\
\text { vezes, apresentava EC }\end{array}$} & $\begin{array}{l}\text { FR (melhora da taquipneia) } \\
\text { Melhora do padrão e ritmo } \\
\text { respiratório } \\
\uparrow S p O 2 \\
\text { AP ( } \uparrow \mathrm{MV} \text { e } \downarrow \text { intensidade dos RA) } \\
\text { Obs: Em uma sessão houve } \\
\text { taquipneia, em outra se auscultou } \\
\text { sibilos expiratórios ao final da ses- } \\
\text { são. E em duas sessões } \downarrow \mathrm{SpO} 2\end{array}$ \\
\hline $03 / 10$ à $31 / 10$ & $\begin{array}{l}\text { FtM: PC, DC,M, ME, DP, CC,AA, } \\
P(P) \text {, estímulo a extensão de } \\
\text { tronco e rotação cervical, } P(D L), \\
P(S), R E, P(4), F N P, A L(A L), A L(I), \\
\text { ES, EVA } \\
\text { FtR: } V C M, G T, E L P r, D A, A F E l, A F E r, \\
B S, C / D, A(T A), A(I C), E F, A\end{array}$ & $\begin{array}{l}\text { Gera } \\
\text { parte }\end{array}$ & $\begin{array}{l}\text { Me G, na maior } \\
\text { coloração MP }\end{array}$ & \multicolumn{2}{|c|}{$\begin{array}{l}\text { FC: Variou de } 56 \text { à } 191 \text { bpm. } \\
\text { Geralmente na faixa de } 110 \text { à } \\
150 \text { bpm; } \\
\text { FR: Variou de } 15 \text { à } 68 \text { rpm Neste } \\
\text { mês, paciente chegava as } \\
\text { sessões com FR mais elevadas, na } \\
\text { faixa de } 35 \text { à } 60 \text { rpm } \\
\text { SpO2: Variou de } 92 \% \text { à } 98 \% \text {. } \\
\text { Geralmente os valores estavam } \\
\text { abaixo de } 95 \% \text { ao início dasessão. } \\
\text { AP: MV } \downarrow \text {, com RD em ambos hmt, } \\
\text { além de RT. Às vezes, apresen- } \\
\text { tava EC. } \\
\text { Obs: Houve dois episódios de } \\
\text { febrículas durante atendimento }\end{array}$} & $\begin{array}{l}\downarrow \text { FR (melhora dataquipneia) } \\
\text { Melhora do padrão e ritmo } \\
\text { respiratório } \\
\uparrow \text { SpO2 } \\
\text { AP ( } \text { ( } \text { intensidade dos RA e } \\
\text { ausência de RA pulmonares } \\
\text { extra-basal) } \\
\text { Obs: Em uma sessão houve } \\
\text { taquipneia e em outras duas } \\
\text { houve } \downarrow \text { SpO2 }\end{array}$ \\
\hline Período & \multicolumn{2}{|l|}{ Aspiração em domicílio } & \multicolumn{2}{|c|}{ Aspecto da secreção } & \multicolumn{2}{|c|}{ Observações } \\
\hline $\begin{array}{l}07 / 09 \text { à } \\
30 / 09\end{array}$ & \multicolumn{2}{|c|}{$\begin{array}{l}176 \text { aspirações no mês, durante } 24 \text { dias. } \\
\text { Média de } 7,3 \text { aspirações/dia }\end{array}$} & \multicolumn{2}{|c|}{$\begin{array}{l}\text { Na maior parte } \mathrm{F} \text { e } \mathrm{C} \text { com alguns episódios } \\
\text { de rolhas. Houve } 3 \text { dias sem que houve } \uparrow \\
\text { quantidade de secreção }\end{array}$} & \multicolumn{2}{|c|}{$\begin{array}{l}\text { Choroso; Irriado; Dificuldade para dormir; } \\
\text { Bastante tosse; } \uparrow \text { secreção; Dificuldade para } \\
\text { respirar }\end{array}$} \\
\hline $\begin{array}{l}01 / 10 \text { à } \\
31 / 10\end{array}$ & \multicolumn{2}{|c|}{$\begin{array}{l}261 \text { aspirações no mês, durante } 31 \text { dias. } \\
\text { Média de } 8,4 \text { aspirações/dia }\end{array}$} & \multicolumn{2}{|c|}{$\begin{array}{l}\text { Nos primeiros } 10 \text { dias caracterizava-se em } \mathrm{P} \text {, } \\
\mathrm{Fe} \mathrm{C} \text {. Porém, houve episódios de } \mathrm{G} \text { e } \uparrow \text { da } \\
\text { viscosidade, até o final do mês }\end{array}$} & \multicolumn{2}{|c|}{$\begin{array}{l}\text { Picos febris (2x); Febre (4x); Febre de difícil } \\
\text { controle (2x); Diagnóstico de pneumonia }\end{array}$} \\
\hline $\begin{array}{l}01 / 11 \text { d̀ } \\
19 / 11\end{array}$ & \multicolumn{2}{|c|}{$\begin{array}{l}167 \text { aspirações no mês, durante } 19 \text { dias. } \\
\text { Média de } 8,7 \text { aspirações/dia }\end{array}$} & \multicolumn{2}{|c|}{$\begin{array}{l}\text { Na maior parte caracterizava-se em F e C. } \\
\text { Poré, houve episódios no início e ao longo do } \\
\text { mês de secreção E }\end{array}$} & \multicolumn{2}{|c|}{ Sem observações feitas pela mãe } \\
\hline
\end{tabular}

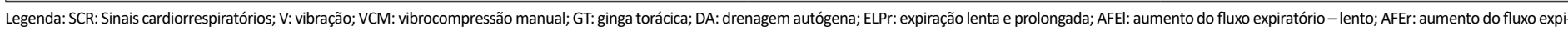

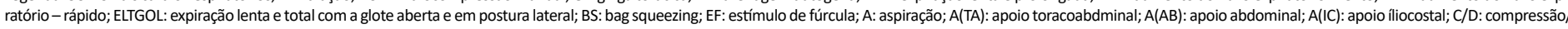

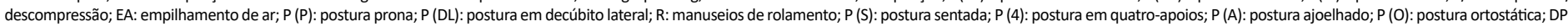

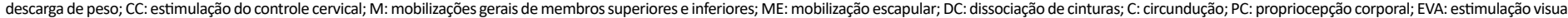

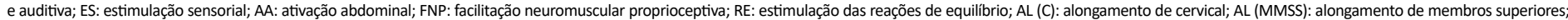

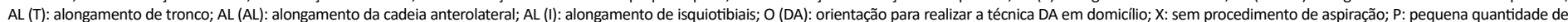

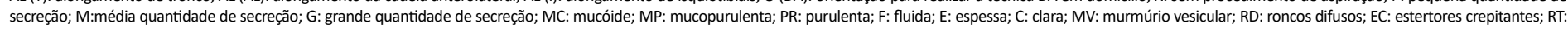
roncos de transmissão; SV: sinais vitais; AV(N): avaliação neurológica; SDR: sinais de desconforto respiratório; $\uparrow:$ Aumento; $\downarrow$ :Diminuição.

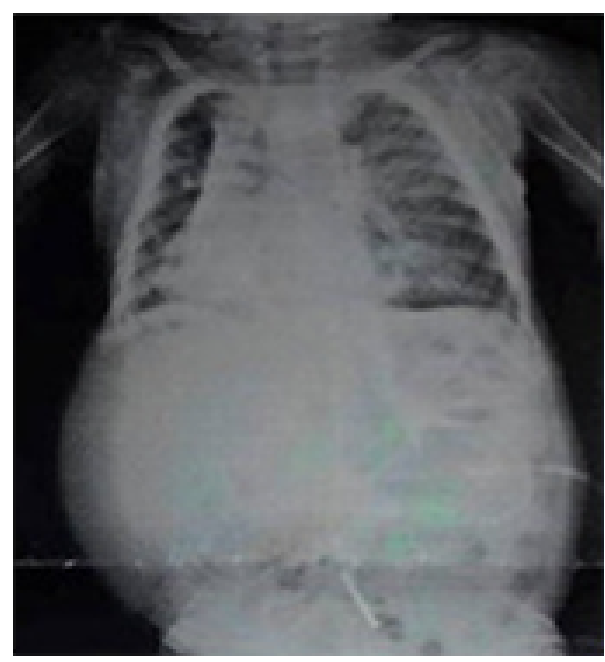

Figura 1. Agosto de 2016

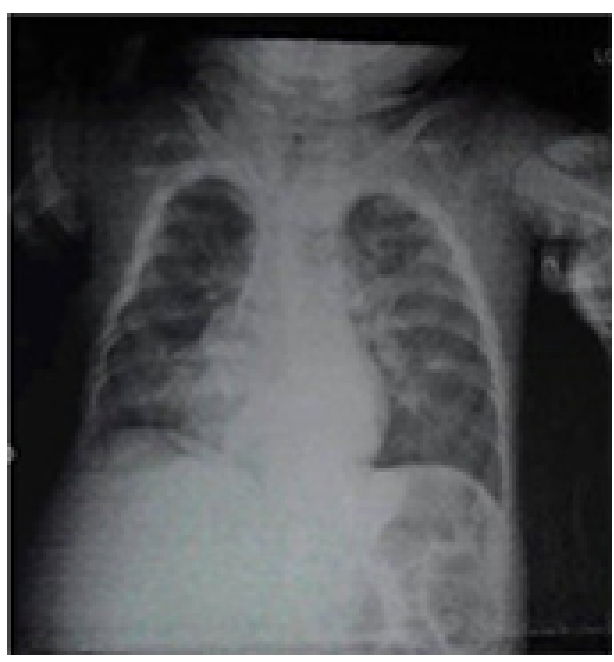

Figura 2. Outubro de 2016 encontrar outros relatos que reforçam a importância dessa terapêutica em síndromes raras, com acometimentos respiratórios e motores, com o objetivo de manter e melhorar o quadro de saúde.

Nesse sentido, Nascimento et al. ${ }^{9}$ realizaram Ft em uma criança com Síndrome de Leigh com o objetivo de melhorar a condição motora, respiratória e, consequentemente, a qualidade de vida, assim como neste presente relato. As sessões descritas nessa publicação foram realizadas $1 \mathrm{x} /$ dia e variavam de 30 à 45 minutos, com as seguintes condutas: mobilizações articulares e alongamentos passivos de MMSS e MMII, dissociações de cinturas, posicionamentos no leito, treinos de rolamento e de equilíbrio. Realizou-se também tapotagem, percussão e vibrocompressão. 
Quadro 4. Evolução do paciente

\begin{tabular}{|c|c|}
\hline FtM & FtR \\
\hline Não houve mudanças quanto a pontuação da GMFM. & $\begin{array}{l}\text { Melhora nos parâmetros cardiorrespiratórios finais: } \mathrm{FC} \mathrm{(dentro} \mathrm{faixa} \mathrm{de} \mathrm{normalidade)} \mathrm{,} \mathrm{FR} \\
\text { (menor número de irpm - melhora da taquipneia), } \mathrm{SpO}_{2} \text { (aumento) }\end{array}$ \\
\hline $\begin{array}{l}\text { Adquiriu maior quantidade e qualidade dos movimentos de membros superiores e } \\
\text { membros inferiores, no entanto, sem conseguir trazer a mão à linha média }\end{array}$ & Melhora no padrão, ritmo e tipo respiratório (quando comparado às sessões iniciais) \\
\hline Melhora do tônus muscular (menor hipotonia quando comparado às sessões iniciais) & $\begin{array}{l}\text { Murmúrio vesicular mais audível, redução dos ruídos adventícios em intensidade e } \\
\text { localização }\end{array}$ \\
\hline Manutenção da escoliose (as deformidades presentes previamente não evoluíram) & Diminuição dos sinais de desconfortos respiratórios, mantendo apenas tiragem intercostal \\
\hline Paciente pareceu mais ativo e menos agitado a cada intervenção & Menor ocorrência de complicações respiratórias e diminuição das crises e internaçõe \\
\hline \multicolumn{2}{|c|}{ Relato da mãe } \\
\hline \multicolumn{2}{|c|}{$\begin{array}{l}\text { "A fisioterapia foi fundamental para ajudar a prolongar a vida do meu filho, ainda mais depois das atelectasias. A fisioterapia sempre trouxe um alívio muito grande, soltava } \\
\text { consideravelmente as secreções, além de auxiliar no alongamento do seu corpinho, já que não se movimentava devido a doença. Meu filho fez uso de vários medicamentos } \\
\text { diariamente, mas sem a ajuda e o profissionalismo dos fisioterapeutas não teria suportado chegar onde chegou. Quando a universidade entrou em férias no final de novembro de } \\
2015, \text { e passou atender uma vez por semana de forma voluntária, teve uma piora considerável e veio falecer dia } 15 \text { de dezembro. Como mãe de um filho que passou por vários } \\
\text { procedimentos invasivos, que lutou a seu modo para viver, afirmo novamente que a fisioterapia foi muito importante." }\end{array}$} \\
\hline
\end{tabular}

Os autores afirmam que o acompanhamento preveniu deformidades, aumentou movimentos de MMSSe MMII, evitou aumento da hipotonia da musculatura axial, melhorou o controle de tronco e cervical, o que contribuiu de forma positiva e impediu agravamentos dos sinais e sintomas. ${ }^{10}$ Em outro estudo, Araújo et al. ${ }^{11}$ relataram a intervenção fisioterapêutica em um acriança de 5 anos com Síndrome de Rett. Aos 2 anos e 5 meses a criança apresentou características da síndrome, sendo elas: hipertonia em MMII, rigidez articular em roda denteada, clônus, movimentos estereotipados, marcha atáxica, escoliose em ' $S$ ', pé equino-varo e incursões respiratórias com pausa.

Atribuiu-se ao acompanhamento fisioterapêutico de 2 meses, com sessões $5 x /$ semana de 1 h e 40 minutos de duração, melhora significativa nas amplitudes de movimentos, padrão ventilatório e quebra do padrão espástico. ${ }^{11}$ Os efeitos da reabilitação cardiorrespiratória em crianças com Síndrome de Prader-Willi foram descritos em uma série de 3 casos, conduzida por Segura et al. ${ }^{12}$ Crianças com idades entre 4 e 5 anos foram submetidas a 3 meses de acompanhamento, total de 28 sessões, $2 x /$ semana e duração de 45 minutos, que resultaram na melhora do quadro geral dos pacientes (parâmetros cardiorrespiratórios, tolerância ao exercício e diminuição do IMC). ${ }^{12}$

Esses estudos citados demonstram que a intervenção fisioterapêutica em casos de síndromes raras está sendo apresentada na literatura em forma de relatos de caso ou série de casos, sendo ausentes as publicações com amostras maiores e ensaios clínicos de qualidade. No entanto, esses registros têm apresentado benefícios do acompanhamento fisioterapêutico, o que indica a importância dessa intervenção, tanto FtM como FtR. Desta forma, o acompanhamento fisioterapêutico parece ser indispensável na SM, pois tem potencial de minimizar sintomas da síndrome, atuando diretamente no DNPM, além da manutenção do quadro respiratório.

Especificamente sobre a FtR, estudos demonstram efeitos positivos com a aplicação de condutas utilizadas no presente relato, em situações como: lactentes cardiopatas submetidos à cirurgia cardíaca e prematuros com baixo peso internados em UTI. ${ }^{6,10}$ A melhora da SpO2, redução da $F R$ e dos SDR, sem alterações no comportamento, reafirmam esses efeitos. Importante ressaltar que durante o acompanhamento fisioterapêutico houve melhora do padrão radiológico global e quase reversão da imagem de atelectasia, o que reforça os benefícios das condutas realizadas neste caso. Atualmente, a FtR atua em âmbito preventivo e tratamento nessa condição de colapso, e relatos de casos na bronquite e na prematuridade, evidenciando benefícios, são identificados. ${ }^{13,14}$

Do ponto de vista motor, sabe-se que a falta de movimento associada aos posicionamentos inadequados, predispõem a modificações morfológicas articulares, musculares e de tecidos conjuntivos..$^{15}$ Desta forma, os relatos de casos mencionados acima, ratificam aimportância da atuação da FtM em diferentes síndromes, como aqui na SM (Quadro 4), trazendo resultados positivos quanto a manutenção do quadro de saúde, prevenção de deformidades, aumento de movimentos e da amplitude, diminuição da hipotonia, quebra de padrões espásticos e melhora no controle neuromuscular.

Além da assistência fisioterapêutica realizada por profissionais, no hospital e ambulatório, a importância da educação e orientação aos pais, para que possam otimizar o tratamento da criança no domicílio, foi conduta adotada no presente relato. ${ }^{7}$ Quando bem orientados, os familiares podem direcionar adequadamente os cuidados à criança e contribuir com o processo de reabilitação em casa. ${ }^{7}$

Apesar do efeito positivo imediato a cada sessão, e pelos reconhecidos benefícios verbalizados pela família e observados pelos profissionais envolvidos, observou-se a progressão da doença, com piora do quadro clínico geral, o que levou o paciente a falecer no início do mês de dezembro. Como já referido anteriormente, a forma clássica da SM possui alterações neurológicas graves, o que contribui com prognóstico fatal nos primeiros anos de vida. ${ }^{1,2,5}$ No entanto, os benefícios da Ft são reconhecidos pela própria família, conforme relato da mãe (Quadro 4).

Vale salientar a necessidade de novos estudos avaliando e controlando a abordagem fisioterapêutica direcionada a essa síndrome rara, uma vez que é ainda escasso material teórico e estudos relacionados ao assunto.

\section{CONCLUSÕES}

O acompanhamento fisioterapêutico de 4 meses, no caso aqui apresentado, demonstrou ter grande importância, pois favoreceu a prevenção de complicações respiratórias, proporcionou maior conforto e impacto positivo na qualidade do sono, comportamento e na vida do paciente. Além disso, a estimulação motora e manutenção do quadro musculoesquelético do paciente assistido impediram agravos e 
deformidades, o que facilitou o manuseio da mãe para transferências e cuidados pessoais no domicílio.

\section{REFERÊNCIAS}

1. Dozza ALCB, Fernandes GD, Yuen CT, Santanna BA, Souza KS, Araújo APQC. Doença de Menkes: relato de caso. Rev Bras Neurol. 2009;45(4):43-7.

2. Kim JH, Lee BH, Kim YM, Choi JH, Kim GH, Cheon $\mathrm{CK}$, et al. Novel mutations and clinical outcomes of copper-histidine therapy in Menkes disease patients. Metab Brain Dis. 2015;30(1):75-81. Doi: http:// dx.doi.org/10.1007/s11011-014-9569-5

3. Costa LS, Pegler SP, Lellis RF, Krebs VL, Robertson $\mathrm{S}$, Morgan T, et al. Menkes disease: importance of diagnosis with molecular analysis in the neonatal period. Rev Assoc Med Bras (1992). 2015;61(5):407-10. Doi: http://dx.doi.org/10.1590/1806-9282.61.05.407

4. Reed UC, Rosemberg S, Diament AJ, Scaff M, Canelas HM, Lefèvre $A B$. Síndrome de menkes: revisão da patogenia a propósito de um caso clínico. Arq Neuro-Psiquiatr. 1984;42(3):262-73. Doi: https://doi.org/10.1590/\$0004282X1984000300010
5. Rodrigues A, Gaspar M, Santos M, Neves N, Lamy $S$, Rendeiro C. Síndrome de Menkes: dois casos clínicos. Acta Pediatr Port. 2002;6(33):445-7.

6. Assumpção $M S$, Gonçalves RM, Ferreira $G L$, Krygierowicz LC, Toews AC, Schivinski CIS. Influência do apoio manual nos parâmetros cardiorrespiratórios, dor e desconforto respiratório em lactentes cardiopatas. Pediatr Mode. 2013;51(10):349-54

7. Russell DJ, Rosenbaum PL, Wright M, Avery LM. Medida da função Motora Grossa. 2 ed. São Paulo: Memnon; 2015.

8. Melo A, Gama GL, Silva Júnior RA, Assunção PL, Tavares JS, Silva MB, et al. Motor function in children with congenital Zika syndrome. Dev Med Child Neurol. 2020;62(2):221-6. Doi: https://doi. org/10.10.1111/dmcn.14227

9. Nascimento $\mathrm{CE}$, Krueger E, Neves EB. Tratamento fisioterápico na síndrome de Leigh: estudo de caso. J Health Sci Inst. 2013;31(3):330-4.

10. Roussenq KR, Scalco JC, Rosa GJ, Honório GJS, Schivinski CIS. Reequilíbrio tóraco-abdominal em recém-nascidos prematuros: efeitos em parâmetros cardiorrespiratórios, no comportamento, na dor e no desconforto respiratório. Acta Fisiatr. 2013;20(3):118-23. Doi: https://doi. org/10.5935/0104-7795.20130019
11. Araújo $A M C$, Nascimento $C M$, Fuziel LC, Braga MVR, Brasil TAC, lannuzzi ML. Tratamento fisioterapêutico ao paciente portador da síndrome de Rett. EFDeportes.com Rev Dig. 2013;18(181).

12. Segura DCA, Nascimento FC, Sarro TCA, Navarro M, Guimarães LF, Orssato L, et. al. Efeitos das técnicas de reabilitação cardiorrespiratória em crianças portadoras da síndrome de Prader-Willi. Arq Ciênc Saúde UNIPAR. 2009;13(2):89-96. Doi: https://doi. org/10.25110/arqsaude.v13i2.2009.3010

13. Fiatt MP, Daher BR, Santos AM. Reversão de atelectasia em recém-nascido prematuro após uma sessão de fisioterapia respiratória - relato de caso. Revista HCPA. 2013;33(3/4):269-73.

14. Gonçalves RM, Albuquerque YP, Ferreira LG, Assumpção MS, Bobbio TG, Schivinski CIS. Relato de caso: intervenção fisioterapêutica na atelectasia. Rev Ciênc Méd Biol. 2013;12(2):253-6. Doi: http://dx.doi. org/10.9771/cmbio.v12i2.6765

15. Pavão SL, Silva FPS, Rocha NAC. Efeito da orientação domiciliar no desempenho funcional de crianças com necessidades especiais. Motricidade. 2011;7(1):21-9. Doi: https://doi.org/10.6063/motricidade.7(1).117 